\section{In defence of CERN}

SIR - May an observer well acquainted with US and European particle physics comment on the report of social scientists Ben Martin and John Irvine on the achievements of CERN, the European High Energy Laboratory in Geneva (Nature 6 September, p.4)? I find this report unfairly biased against CERN.

When European high-energy accelerator research on a large scale began in 1960 , CERN was a completely novel social experiment. There was almost no tradition of "big science" in Europe, and no experience of international scientific management. Nevertheless, within less than 24 years, CERN had developed into the world's leading laboratory in this field.

Why then do Martin and Irvine consider CERN's performance disappointing? They concentrate their attention on sensational peak discoveries, in which respect CERN has contributed only in recent decades. But science does not consist of discoveries that make headlines in newspapers. The bulk of science consists of a broad front of painstaking investigations, leading to ever deeper insights. They are the soil from which striking discoveries can develop. In this respect, CERN has made many contributions to which Martin and Irvine give only passing attention.

It is true that some of the peak discoveries, in particular the so-called $c$ (charm) and $b$ (bottom) quarks, could have been found at the intersecting storage rings (ISR) at CERN. Other "sensational" discoveries, the violation of parity, the $\Omega$-minus and the two types of neutrinos, which CERN is blamed for missing, were made in the United States before or at a time when research had barely started at CERN. But even if one insists on counting the lucky events of that type, CERN did not fare so badly during the second half of its research period. It discovered a new form of radioactivity, the so-called neutral currents, the well-advertised quanta of the weak interaction and probably a sixth quark ( $t$, for top) as well.

Martin and Irvine ascribe the latest achievements to a recent change of spirit due to the present director. It is true that Herwig Schopper vigorously supported the last phases of the conversion of the super proton synchrotron (SPS) as a protonantiproton collider, and the subsequent successful research. However, Martin and Irvine do not seem to appreciate that the conversion of an accelerator and the preparation of such giant experiments take much longer than the three and a half years of Schopper's directorship. The daring spirit and the technical inventiveness that have led to these achievements date from much earlier times. They are a proof of the opportunities given to individual scientists, which Martin and Irvine consider to have been too restricted at CERN. They also show a steady growth of this institution towards its present world supremacy, that started long ago.

The comparison with Fermilab in the United States is unfairly presented. True enough, several great discoveries were made at that laboratory, such as the existence of a fifth quark. But it was the CERN accelerator that was able to produce sharp and intense muon and neutrino beams with which the so-called structure functions of protons and neutrons were determined with astounding accuracy. Moreover, the important "EMC-effect" was discovered, which has led to a new view of the role of quarks in the atomic nucleus. One of the main purposes of those accelerators was the production of usable secondary muon and neutrino beams, in which CERN was much more successful than Fermilab inspite of the latter's fouryear lead. Why do Martin and Irvine not appreciate these successes? Why do they consider them to be dull physics?

CERN's contributions to the physics of nuclear structure are almost completely neglected in Martin and Irvine's report. CERN has the most advanced isotope separation device (Isolde) attached to its synchro-cyclotron, where many new unstable nuclei have been discovered and studied. Recently, a low-energy source for antiprotons was constructed (LEAR) which provides unique opportunities for the study of antiproton interactions.

The success of CERN is based to a large extent upon a circumstance that has been a surprise for people who have observed the development of physics in the past half century. The United States was assumed to the superior in engineering and instrumentation, as demonstrated by the development in the 1930s of cyclotrons, synchrotrons, linear accelerators, bubble chambers and so on. But the quality of the engineerphysicists at CERN turned out to be at least as high. They succeeded in constructing accelerators of unusual reliability and flexibility under the leadership of the late Sir John Adams. Some have ascribed this to over-conservative design, but it helped greatly in the exploitation of the machines. Thus it allowed the luminosity of the intersecting storage rings to reach more than ten times the design value, and it facilitated the production of the intense muon and neutrino beams. Finally, it made possible the fast conversion of the $400 \mathrm{GeV}$ accelerator into a proton-antiproton collider, the success of which hung on one of the imaginative devices invented and implemented at CERN by Simon Van der Meer - the so-called stochastic cooling of the antiproton beam, destined to be used in future colliders everywhere in the world.

Furthermore, CERN engineer-physicists led by Kjell Johnson constructed the intersecting storage rings at a time when laboratories in the United States did not dare to take on this difficult task. CERN designed and built the first two colliders for protons, the ISR and the protonantiproton collider in the SPS tunnel. The effect has been to pioneer a new style of experimentation which has now been taken up by other centres of particle research all over the world. CERN continues the tradition with the large electron-positron collider LEP.

The Martin-Irvine report does not make much of these achievements and also all but ignores the many new ideas for instrumentation spawned at CERN, such as the multiwire proportional chamber and the vacuum ultraviolet imaging device. Both innovations are not only significant advances in detecting particles, but have important applications in medicine and in other sciences.

High-energy physics is an international endeavour, more so than most other scientific activities. There is a constant exchange of physicists and engineers between CERN, the United States and other countries. It does not make much sense to consider progress in this field as a competition between two continents. It is a collective achievement of all participating nations. The CERN effort has contributed essential ingredients. Particle physics would not be what it is without CERN's impetus, without its technical innovations and without its studies and discoveries, even if some of them have not made newspaper headlines.

Last and by no means least, CERN represents the United States of Europe in fundamental physics. It became an active symbol of the spirit of European unity. That is no mean achievement. It serves as the University of Europe for high-energy physics, and brings together the many individual universities in Europe in scientific collaborations of unprecedented magnitude and complexity often involving also universities and institutes of the United States, Eastern Europe and Asia.

In spite of the obvious difficulties which such international collaborations entail, CERN has developed into the foremost high-energy laboratory of the world, as is strikingly confirmed by the recent US panel report on New Facilities for the US High Energy Physics Program, in which it was stated that "the European facilities frequently provide a better level of support and/or a larger number of opportunities than the American equivalents".

How can such an institution be given "Poor Marks for Enterprise"?

VICTOR F. WEISSKOPF

Massachusetts Institute of Technology, Cambridge, Massachusetts 02139, USA

Victor Weisskopf was director of CERN from 1961 to 1965.

SIR - Robert Walgate's enthusiasm (see Nature 6 September, p.4) for the assessment of CERN carried out by two social scientists from Sussex, Irvine and Martin, is misplaced. Most would agree with one conclusion reached by Martin and Irvine, 